Jpn. J. Infect. Dis., 74, 352-358, 2021

Original Article

\title{
Panton-Valentine Leukocidin Induces Cytokine Release and Cytotoxicity Mediated by the C5a Receptor on Rabbit Alveolar Macrophages
}

\author{
Shinya Harada ${ }^{1,2}$, Hayato Kawada ${ }^{3}$, Shotaro Maehana ${ }^{5}$, Hidehito Matsui ${ }^{4}$, Makoto Kubo ${ }^{6}$, Fumiaki Kojima ${ }^{7}$, \\ Hidero Kitasato ${ }^{5}$, and Masato Katagiri ${ }^{1,2,8 *}$ \\ ${ }^{1}$ Kitasato University Graduate School of Medical Sciences, Kanagawa; \\ ${ }^{2}$ Department of Respiratory Medicine, Kitasato University Hospital, Kanagawa; \\ ${ }^{3}$ Department of Parasitology and Tropical Medicine, Kitasato University School of Medicine, Kanagawa; \\ ${ }^{4}$ Omura Satoshi Memorial Institute, Kitasato University, Tokyo; and ${ }^{5}$ Department of Microbiology, \\ ${ }^{6}$ Division of Immunology II, ${ }^{7}$ Department of Pharmacology, and \\ ${ }^{8}$ Department of Clinical Physiology, Kitasato University School of Allied Health Sciences, Kanagawa, Japan
}

\begin{abstract}
SUMMARY: Necrotizing pneumonia caused by Panton-Valentine leukocidin (PVL)-positive community-acquired methicillin-resistant Staphylococcus aureus (CA-MRSA) has high mortality rates and is currently a serious clinical issue. PVL is a two-component toxin (LukS-PV and LukF-PV). It can cause necrosis in target cells by forming pores consisting of an octamer comprised of LukS-PV and LukF-PV. However, considering the specificity of PVL towards several target cells and species, the specific effect of PVL remains controversial. Therefore, we focused on necrotizing pneumonia caused by PVL-positive $S$. aureus and clarified the effect of PVL on alveolar macrophages, which play a central role in innate immunity in the alveolar space. We constructed recombinant PVL (rPVL) components and stimulated alveolar macrophages isolated from rabbits to evaluate cytotoxicity and pro-inflammatory cytokine release. Recombinant LukS-PV (rLukS-PV), but not recombinant LukF-PV (rLukF-PV), induced pro-inflammatory cytokine release. Specifically, tumor necrosis factor (TNF)- $\alpha$ release was mediated by the $\mathrm{C} 5 \mathrm{a}$ receptor $(\mathrm{C} 5 \mathrm{aR})$ expressed on rabbit alveolar macrophages, and the toxicity of rPVL, consisting of rLukS-PV and rLukF-PV, towards rabbit alveolar macrophages was mediated by the same receptor. Overall, our findings shed light on the C5aR-mediated cytotoxic effect of PVL on alveolar macrophages, which may be useful for understanding the mechanism of necrotizing pneumonia caused by PVL.
\end{abstract}

\section{INTRODUCTION}

Staphylococcus aureus invades the body through damaged skin and mucous membrane and produces various toxins, which may result in infections such as skin and soft tissue infections, pneumonia, infective endocarditis, and osteomyelitis. Methicillin-resistant S. aureus (MRSA), first reported in 1961, causes nosocomial infection (1). Various reports on MRSA were subsequently published in the 1960s and 1970s (2). Hospital-acquired MRSA appeared in the 1980s and community-acquired MRSA (CA-MRSA) appeared in the 1990s (3). Severe infections due to CA-MRSA have been reported in western countries, Australia, and

Received August 4, 2020. Accepted December 14, 2020. J-STAGE Advance Publication January 29, 2021.

DOI: 10.7883 /yoken.JJID.2020.657

*Corresponding author: Mailing address: Kitasato University School of Allied Health Sciences, Kitasato 1-15-1, Minamiku, Sagamihara, Kanagawa 252-0373, Japan. Tel: +8142-778-8111, Fax: +81-42-778-6412, E-mail: mkata@ kitasato-u.ac.jp
Japan (4-6). Particularly, the MRSA USA300 strain causes necrotizing pneumonia (7) and sepsis (8). An important characteristic of CA-MRSA, including the USA300 strain, is a high Panton-Valentine leukocidin (PVL) retention rate (9). Mortality rates are higher in pneumonia caused by PVL-positive MRSA compared with that caused by PVL-negative MRSA (10); thus, PVL is a major factor that affects the severity of MRSA infection.

PVL, a two-component toxin consisting of LukS-PV and LukF-PV, induces cell death in human neutrophils, monocytes (11), and alveolar macrophages (12). First, LukS-PV binds to receptors on target cells (13) and forms a dimer with LukF-PV (14). Thereafter, the oligomerization of LukS-PV and LukF-PV occurs, and the octamer forms a pore on the target cell membrane $(15,16)$. Finally, the death of target cells is induced by the loss of homeostasis (17). LukS-PV binds to the C5a receptor (C5aR) on neutrophils and macrophages $(18,19)$. However, the trigger for PVL action remains unknown for other target cells.

Holzinger et al. reported that pore formation induces PVL to activate the nucleotide-binding domain leucine- 
rich repeat-containing gene family and pyrin domaincontaining 3 protein inflammasome, leading to the release of interleukin (IL)-1 $\beta$ from target cells (20). However, Zivkovic et al. showed that target cells release TNF- $\alpha$ independently of PVL-induced pore formation (21). Considering the action of the various toxins produced by MRSA and the complex host specificity of PVL itself $(11,19)$, the specific effect of PVL remains unclear.

It is necessary to clarify the effect of PVL in necrotizing pneumonia. Alveolar macrophages play a key role in the early stage of lung infection and regulate various immune cells. Here, we evaluated the cytotoxicity and inflammatory response induced by PVL and its components, LukS-PV and LukF-PV, using primary cultured alveolar macrophages from rabbits.

\section{MATERIALS AND METHODS}

Reagents: PMX205 (Tocris Bioscience, Bristol, UK), a blocker of C5a binding (22), was used in C5a receptor inhibition experiments. Lipopolysaccharide (LPS, 100 ng/mL; Sigma-Aldrich, St. Louis, MO, USA) was used as a positive control.

Cloning of rLukS-PV and rLukF-PV: Recombinant proteins were prepared by modification of a previously described method (23). In brief, recombinant $6 \times$ Histagged rLukS-PV and rLukF-PV proteins were expressed using the pRSET vector (Thermo Fisher Scientific, Waltham, MA, USA). The sequences of $l u k S$ and $l u k F$, derived from the MRSA USA300 strain and attached to the recognition sequences of restriction enzymes, were produced by MBL Co., Ltd. (Aichi, Japan). These sequences were incorporated into pRSET digested with BamHI (Takara, Shiga, Japan) and XhoI (Takara) or HindIII (Takara), respectively. LukS-PVpRSET, LukF-PV-pRSET, and empty vector (EV)pRSET were transformed into competent Escherichia coli DH5 $\alpha$ cells (Thermo Fisher Scientific). The sequences of $l u k S$ and $l u k F$ were confirmed using the genomic DNA sequencing service of Eurofins (Luxembourg, Grand Duchy of Luxembourg).

Preparation of recombinant protein: LukSPV-pRSET, LukF-PV-pRSET, and EV-pRSET were transformed into E. coli BL21(DE3) cells (Thermo Fisher Scientific). The transformed cells were cultured with isopropyl- $\beta$-D-thiogalactoside (Takara) for $2 \mathrm{~h}$ at $37^{\circ} \mathrm{C}$ to express recombinant proteins. The bacterial pellet was resuspended in $1 \times$ phosphate-buffered saline (PBS) (-) and sonicated. The recombinant proteins were purified using the TALON cobalt affinity resin (Takara). The solvent was replaced with PBS (-) using a dialysis membrane (Spectrum Laboratories, New Brunswick, NJ, USA). The removal of LPS was performed twice using DetoxiGel columns (Thermo Fisher Scientific), and the concentration of LPS was confirmed to be less than $0.005 \mathrm{EU} / \mathrm{mL}$ using a Limulus Color KY test kit (FUJIFILM Wako Pure Chemical, Tokyo, Japan). The concentrations of the recombinant proteins were measured using the NanoDrop ${ }^{\mathrm{TM}}$ Lite spectrophotometer (Thermo Fisher Scientific).

The expression of recombinant LukS-PV (rLukSPV), LukF-PV (rLukF-PV), and EV (rEV) was confirmed by sodium dodecyl sulfate-polyacrylamide gel electrophoresis (SDS-PAGE) and western blotting (WB). $r E V$ is a solvent including a $6 \times$ His-tagged peptide, which was derived from E. coli BL21(DE3) transformed with EV-pRSET obtained through the purification process described above. For WB, rLukS-PV, rLukF-PV, and rEV were blotted onto a polyvinylidene fluoride membrane (Immobilon-P Transfer Membrane ${ }^{\circledR}$; Merck, Darmstadt, Germany) and detected using the Anti $6 \times$ Histidine monoclonal antibody (FUJIFILM Wako Pure Chemical) and horseradish peroxidase (HRP)-conjugated goat antimouse IgG (BioLegend, San Diego, CA, USA). Protein bands were visualized on an X-ray film (FUJIFILM, Tokyo, Japan) after incubation with a chemiluminescent substrate, ECL (GE Healthcare, Little Chalfont, UK). The recombinant proteins were mixed with glycerin $(1: 1 \mathrm{v} / \mathrm{v})$ to prevent aggregation during freezing and thawing. Finally, rLukS-PV and rLukF-PV were mixed $(1: 1 \mathrm{v} / \mathrm{v})$ to obtain recombinant PVL (rPVL). In all toxin stimulation experiments, the first recombinant protein aliquot was used.

Recovery of rabbit alveolar macrophages: Alveolar macrophages were isolated from rabbit lungs by modification of a previously described method (24). In brief, New Zealand white rabbits (female; body weight, $2.82 \pm 0.06 \mathrm{~kg}$; age, $14.71 \pm 0.53$ weeks; $n=14$ ) were anesthetized with isoflurane and pentobarbital and sacrificed by exsanguination. Bronchoalveolar lavage was performed thrice with $50 \mathrm{~mL}$ of sterile saline (Fuso, Osaka, Japan) through an endotracheal tube. The bronchoalveolar lavage fluid (BALF) was centrifuged, and cell pellets were resuspended in RPMI1640 (SigmaAldrich) containing 10\% FBS (Sigma-Aldrich) followed by incubation for $2 \mathrm{~h}$ at $37^{\circ} \mathrm{C}$ with $5 \% \mathrm{CO}_{2}$. After incubation, adherent cells were recovered.

All procedures were approved by the Animal Experimentation and Ethics Committee of Kitasato University School of Allied Health Sciences (Approval number: 18-53).

Rabbit alveolar macrophage culture, stimulation, and C5aR inhibition: Rabbit alveolar macrophages were cultured overnight in RPMI1640 containing $10 \%$ FBS (Sigma-Aldrich) by seeding $1 \times 10^{5}$ cells into each well in tissue culture plates (IWAKI, Shizuoka, Japan). In toxin stimulation experiments, the culture medium was replaced, and alveolar macrophages were stimulated with rLukS-PV, rLukF-PV, rPVL, or rEV at a final concentration of 5 and $100 \mathrm{nM} ; 5$ and $100 \mathrm{nM}$ rPVL comprised $5 \mathrm{nM}$ rLukS-PV + $5 \mathrm{nM}$ rLukF-PV and $100 \mathrm{nM}$ rLukS-PV + 100 nM rLukF-PV, respectively. To measure the levels of inflammatory cytokines, alveolar macrophages were also stimulated with LPS $(100 \mathrm{ng} / \mathrm{mL})$ as a positive control. After stimulation for $24 \mathrm{~h}$, the cells were used for the evaluation of cytotoxicity, and the culture medium was used for the measurement of pro-inflammatory cytokines. In C5aR inhibition experiments, rabbit alveolar macrophages were pretreated with PMX205 for 15 min before stimulation with recombinant proteins.

Cytotoxicity assay: Cytotoxicity towards alveolar macrophages was evaluated using Cell Proliferation Kit I (MTT) (Merck). The absorbance was measured using a microplate reader (measurement wavelength: $570 \mathrm{~nm}$; reference wavelength: $655 \mathrm{~nm}$ ) (Benchmark; Bio-Rad, 
Hercules, CA, USA).

Measurement of pro-inflammatory cytokines: Pro-inflammatory cytokines in the culture supernatant were measured by enzyme-linked immunosorbent assay (ELISA). TNF- $\alpha$ and IL-6 were evaluated with Rabbit TNF-alpha and Rabbit IL-6 DuoSet ELISA kits (R\&D Systems, Minneapolis, MN, USA), and IL-8 and IL-1 $\beta$ were evaluated with a Rabbit ELISA kit (RayBiotech, Peachtree Corners, GA, USA). To standardize the difference in the pro-inflammatory responses of each group, cytokine concentrations were normalized by dividing the value obtained with each toxin by that obtained in LPS-stimulated macrophages, and the results are shown as percentages.

Confirmation of C5aR expression: The expression of $\mathrm{C} 5 \mathrm{aR}$ in rabbit alveolar macrophages was confirmed by WB. Proteins were extracted from cultured cells by incubation in lysis buffer $(20 \mathrm{mM}$ Tris, $0.25 \mathrm{M}$ sucrose, $1 \%$ Triton, $10 \mathrm{mM}$ EGTA, $2 \mathrm{mM}$ EDTA, $\mathrm{pH}$ 8.2) for $1 \mathrm{~h}$ on ice. C5aR was detected using human C5aR monoclonal antibody (clone S5/1; Hycult ${ }^{\circledR}$ Biotech, Uden, The Netherlands) and HRP-conjugated goat antimouse IgG (BioLegend).

Statistical analysis: When comparing three or more groups, significant differences were determined by oneway analysis of variance. To compare the data between two groups, a $t$-test was performed assuming that the F-test showed equal variance. EZR (Saitama Medical Center, Jichi Medical University, Saitama, Japan) was used for statistical analysis. All values are expressed as the mean $\pm \mathrm{SE}$. A p value $<0.05$ was considered statistically significant. When the concentration was lower than the detection sensitivity in the measurement of pro-inflammatory cytokines, statistical analysis was performed by substituting the lower limit of detection of each ELISA kit (TNF- $\alpha: 31.3 \mathrm{pg} / \mathrm{mL}$; IL-6: $12.5 \mathrm{pg} / \mathrm{mL}$; IL-1 $\beta$ : $49.15 \mathrm{pg} / \mathrm{mL})$.

\section{RESULTS}

Purification of recombinant proteins and recovery of alveolar rabbit macrophages: Bands corresponding to the molecular weights of LukS-PV and LukF-PV (32 and $34 \mathrm{kDa}$, respectively) (17) were confirmed by SDS-PAGE (Fig. 1A) and WB (Fig. 1B). No band was observed in the rEV lane (Fig. 1B).

The purity of alveolar macrophages was evaluated morphologically by May-Giemsa staining and immunologically by measuring the expression level of CD11b. Approximately $89 \%$ of the cells recovered from the BALF were morphologically confirmed to be alveolar macrophages (data not shown). Furthermore, CD11b was stained with fluorochrome-conjugated monoclonal CD11b antibody (phycoerythrin; PE; BioLegend) and analyzed by flow cytometry using the MACSQuant flow cytometer (Miltenyi Biotec $\mathrm{GmbH}$, Bergisch Gladbach, Germany). Cells were gated for forward/side scatter, and the CD11b-positive population was designated as alveolar macrophages. Approximately $88 \%$ of cells were CD11b-positive (Fig. 1C).

Cytotoxicity of rPVL towards rabbit alveolar macrophages: The cytotoxicity of rPVL, rLukS-PV, rLukF-PV, and rEV was evaluated by MTT assay. The viability of rPVL-stimulated alveolar macrophages
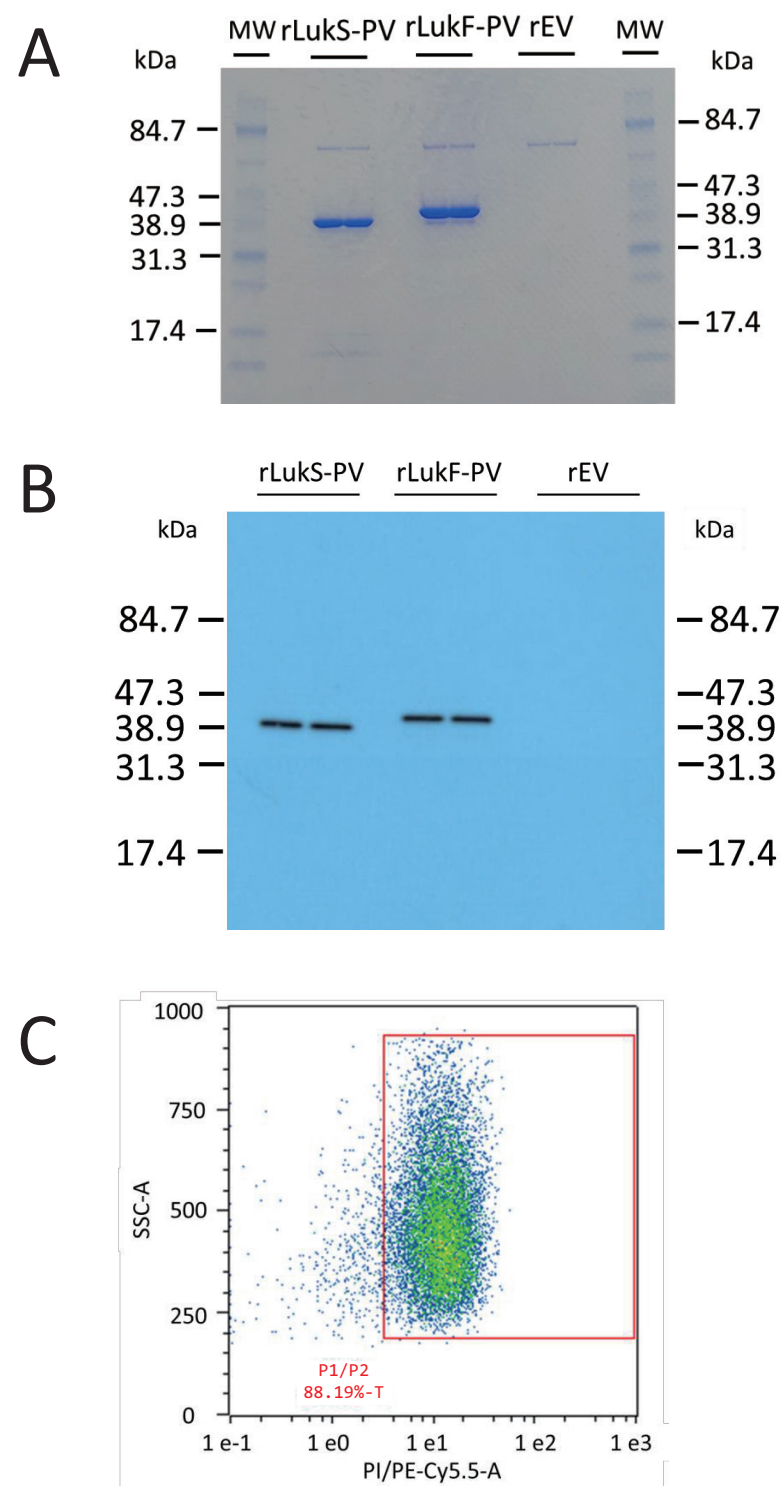

Fig. 1. (Color online) Confirmation of recombinant protein expression and alveolar macrophage purity. Recombinant proteins extracted from $E$. coli were confirmed by (A) sodium dodecyl sulfate-polyacrylamide gel electrophoresis and (B) western blotting. (C) Cells gated by forward and side scattered light were fluorescently stained with CD11b antibody. Approximately $88 \%$ of the cells were stained, with CD11bpositive cells defined as alveolar macrophages.

was significantly reduced in a dose-dependent manner compared with that of unstimulated alveolar macrophages; however, this was not observed with $\mathrm{rEV}$ and single components (Fig. 2). These results indicated that rPVL oligomerization may be necessary to exert cytotoxic effects on alveolar macrophages.

LukS-PV-induced release of inflammatory cytokines from rabbit alveolar macrophages: Several key pro-inflammatory cytokines such as TNF- $\alpha$, IL- 6 , IL- 8 , and IL- $1 \beta$ were released from alveolar macrophages stimulated with rPVL, rLukSPV, rLukF-PV, and rEV (Table 1). The levels of all proinflammatory cytokines were significantly increased by stimulation with rLukS-PV in a concentrationdependent manner. However, compared with the 


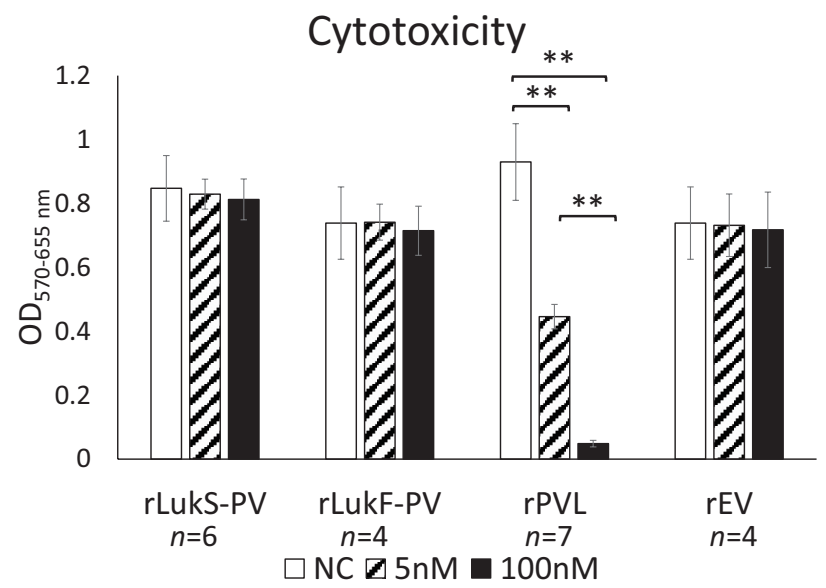

Fig. 2. Evaluation of cytotoxicity to alveolar macrophages by recombinant proteins. Alveolar macrophages $\left(1 \times 10^{5} /\right.$ well $)$ were stimulated with 5 and $100 \mathrm{nM}$ rLukS-PV, rLukF-PV, rPVL (total concentration), and $\mathrm{rEV}$ for $24 \mathrm{~h}$, and cytotoxicity was evaluated by MTT assay. Results represent the means \pm SE. $\mathrm{NC}$, Negative control (unstimulated alveolar macrophages). $n$ indicates the number of rabbits. $* * P<0.01$.

Table 1. PVL and its components-induced inflammatory cytokine release from rabbit alveolar macrophages

\begin{tabular}{|c|c|c|c|c|c|c|c|c|c|c|}
\hline & \multicolumn{2}{|c|}{ rLukS-PV } & \multicolumn{2}{|c|}{ rLukF-PV } & \multicolumn{2}{|c|}{ rPVL } & \multicolumn{2}{|c|}{$\mathrm{rEV}$} & \multirow{2}{*}{ LPS } & \multirow{2}{*}{$\mathrm{NC}$} \\
\hline & $5 \mathrm{nM}$ & $100 \mathrm{nM}$ & $5 \mathrm{nM}$ & $100 \mathrm{nM}$ & $5 \mathrm{nM}$ & $100 \mathrm{nM}$ & $5 \mathrm{nM}$ & 100nM & & \\
\hline TNF- $\alpha$ & $\begin{array}{c}197.99 \\
\pm \\
46.69\end{array}$ & $\begin{array}{c}3,187.98 \\
\pm \\
988.13\end{array}$ & $\begin{array}{c}44.55 \\
\pm \\
10.52\end{array}$ & $\begin{array}{c}228.90 \\
\pm \\
101.53\end{array}$ & $\begin{array}{c}253.19 \\
\pm \\
109.64\end{array}$ & ND & $\begin{array}{c}115.72 \\
\pm \\
67.64\end{array}$ & $\begin{array}{c}142.60 \\
\pm \\
87.64\end{array}$ & $\begin{array}{c}1,340.64 \\
\pm \\
337.58\end{array}$ & $\begin{array}{c}89.39 \\
\pm \\
48.52\end{array}$ \\
\hline IL-6 & $\begin{array}{c}106.66 \\
\pm \\
57.21\end{array}$ & $\begin{array}{c}2,207.73 \\
\pm \\
650.22\end{array}$ & $\begin{array}{c}41.37 \\
\pm \\
8.10\end{array}$ & $\begin{array}{c}121.13 \\
\pm \\
33.00\end{array}$ & $\begin{array}{c}82.63 \\
\pm \\
50.32\end{array}$ & $\begin{array}{c}13.92 \\
\pm \\
1.42\end{array}$ & $\begin{array}{c}36.64 \\
\pm \\
14.78\end{array}$ & $\begin{array}{c}65.04 \\
\pm \\
13.23\end{array}$ & $\begin{array}{c}2,465.02 \\
\pm \\
264.50\end{array}$ & $\begin{array}{c}29.84 \\
\pm \\
5.50\end{array}$ \\
\hline IL-8 & $\begin{array}{c}12,689.29 \\
\pm \\
3,907.33\end{array}$ & $\begin{array}{c}26,515.40 \\
\pm \\
6,191.02\end{array}$ & $\begin{array}{c}5,428.13 \\
\pm \\
2,577.26\end{array}$ & $\begin{array}{c}9,893.73 \\
\pm \\
3,037.68\end{array}$ & $\begin{array}{c}7,220.43 \\
\pm \\
2,736.27\end{array}$ & $\begin{array}{c}767.00 \\
\pm \\
351.38\end{array}$ & $\begin{array}{c}5,880.28 \\
\pm \\
2,966.66\end{array}$ & $\begin{array}{c}7,267.63 \\
\pm \\
3,605.24\end{array}$ & $\begin{array}{c}34,232.79 \\
\pm \\
11,974.81\end{array}$ & $\begin{array}{c}5,673.05 \\
\pm \\
3,045.08\end{array}$ \\
\hline IL- $1 \beta$ & $\begin{array}{c}257.01 \\
\pm \\
118.20\end{array}$ & $\begin{array}{c}651.46 \\
\pm \\
271.47\end{array}$ & $\begin{array}{c}196.24 \\
\pm \\
74.93\end{array}$ & $\begin{array}{c}254.84 \\
\pm \\
112.60\end{array}$ & $\begin{array}{c}146.43 \\
\pm \\
60.61\end{array}$ & $\begin{array}{c}64.56 \\
\pm \\
15.41\end{array}$ & $\begin{array}{c}211.94 \\
\pm \\
85.69\end{array}$ & $\begin{array}{c}242.68 \\
\pm \\
101.49\end{array}$ & $\begin{array}{c}587.65 \\
\pm \\
243.32\end{array}$ & $\begin{array}{c}184.85 \\
\pm \\
66.64\end{array}$ \\
\hline
\end{tabular}

$(\mathrm{pg} / \mathrm{mL})$

negative control (NC), rLukF-PV and rPVL showed no significant difference in cytokine release (Fig. 3). Therefore, rLukS-PV may trigger pro-inflammatory responses in alveolar macrophages.

C5aR-mediated alveolar macrophage cytotoxicity induced by rPVL: The expression of $\mathrm{C} 5 \mathrm{aR}$ on rabbit alveolar macrophages was confirmed (Fig. 4A). To determine whether C5aR is necessary for PVL-induced cytotoxicity, we inhibited C5aR using PMX205. The reduced viability of $5 \mathrm{nM}$ rPVL-stimulated cells was significantly abrogated by pretreatment with PMX205, indicating the vital role of $\mathrm{C} 5 \mathrm{aR}$ in $\mathrm{rPVL}$-induced cytotoxicity (Fig. 4B). In the group stimulated with $100 \mathrm{nM} \mathrm{rPVL}$, there was no difference in cell survival regardless of pretreatment with PMX205 (data not shown), indicating the contribution of other factors besides C5aR to cytotoxicity.

rLukS-PV-induced release of TNF- $\alpha$ via binding to C5aR: PMX205 caused a decrease in TNF- $\alpha$ by inhibiting C5aR during rLukS-PV stimulation (Fig. 4C). When pretreated with $100 \mu \mathrm{M}$ PMX205, TNF- $\alpha$ release from $5 \mathrm{nM}$ rLukS-PV-stimulated cells was decreased from $4.0 \%$ to a level below the lower limit of detection. Similarly, the level of TNF- $\alpha$ released from $100 \mathrm{nM}$ rLukS-PV-stimulated cells was reduced from $128.0 \%$ to $32.2 \%$ following pretreatment.

\section{DISCUSSION}

It is important to understand the mechanism of bacterial pneumonia caused by PVL. As alveolar macrophages are the major immune cells in normal alveoli, we focused on the alveolar space in the early stages of bacterial pneumonia.

The results showed that LukS-PV alone induced the release of inflammatory cytokines from alveolar macrophages, and LukS-PV and LukF-PV formed membrane pores on the surface of alveolar macrophages, leading to cytotoxicity mediated by C5aR.

We found that PVL-induced cytotoxicity towards alveolar macrophages was mediated by C5aR. Zivkovic 

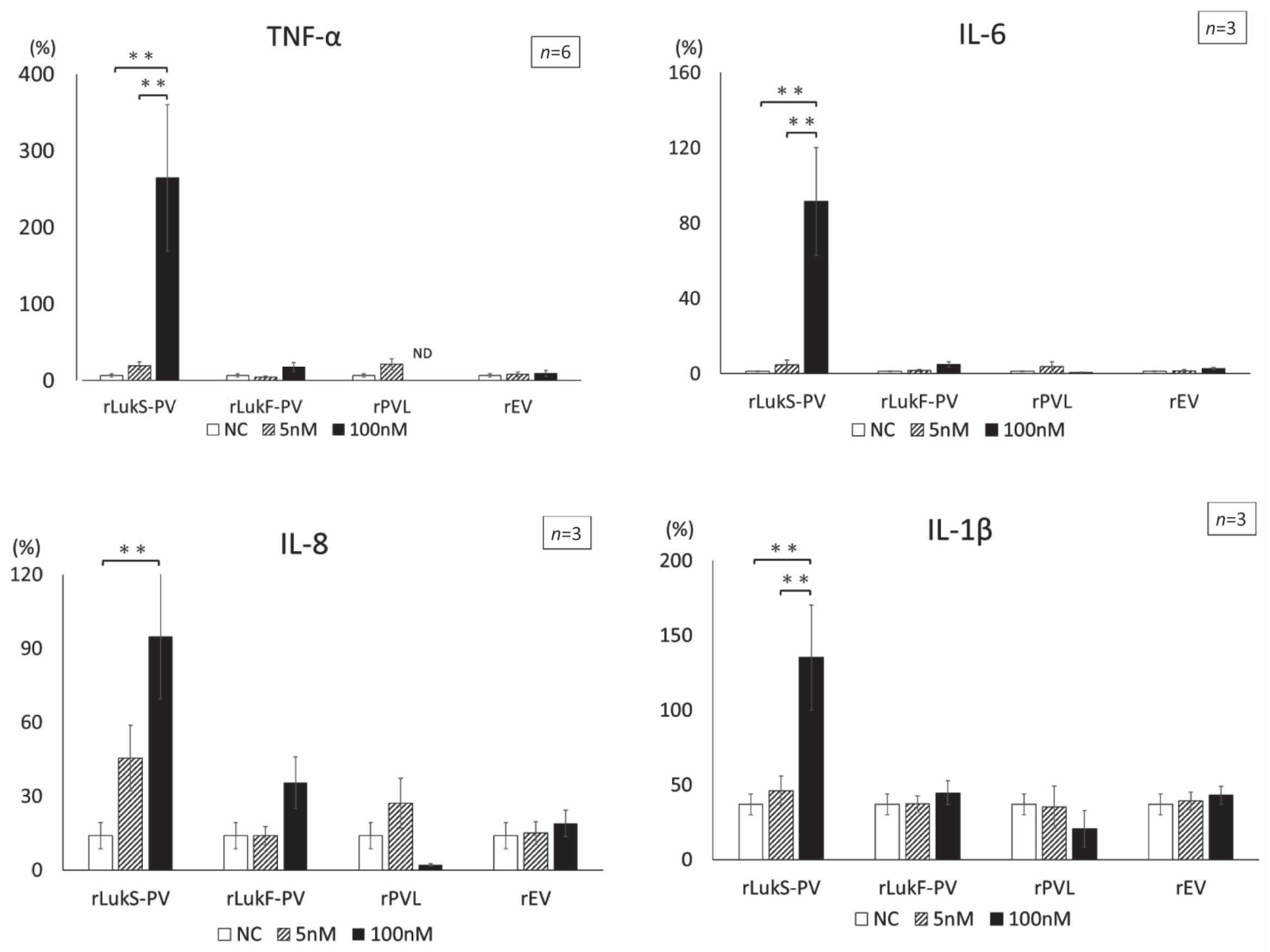

Fig. 3. Cytokine production after stimulation with each recombinant protein. Alveolar macrophages $\left(1 \times 10^{5} /\right.$ well $)$ were stimulated with 5 or $100 \mathrm{nM}$ rLukS-PV, rLukF-PV, rPVL (total concentration), or rEV, $100 \mathrm{ng} / \mathrm{mL}$ LPS (positive control, PC), for $24 \mathrm{~h}$, or were not stimulated (negative control, NC), and each cytokine in the supernatant was measured by ELISA. Results represent the means \pm SE. Significant differences among rLukS-PV, rLukF-PV, rPVL, rEV, and NC were statistically analyzed. $\mathrm{n}$ indicates the number of rabbits. ${ }^{* *} P<0.01$.

et al. (21) and Wu et al. (12) reported that PVL was cytotoxic towards primary cultured human alveolar macrophages; however, the receptor for PVL remains unknown. Spaan et al. (19) reported the involvement of C5aR in PVL cytotoxicity towards human primary cultured neutrophils. However, the induction of local immunity in the alveolar space cannot be fully understood by elucidating only the effect of PVL on neutrophils. Our results shed light on the effect of PVL on rabbit alveolar macrophages and could be useful for understanding the pathophysiology of PVL.

Alveolar macrophages are integral components in early immunity as they recognize exogenous antigens and release inflammatory cytokines such as TNF- $\alpha$ (25). PVL can induce the release of inflammatory cytokines from human neutrophils $(20,21,23)$ and monocytes (20). However, Wu et al. (12) reported that TNF- $\alpha$ was not released from primary cultured human alveolar macrophages at low concentrations of PVL. In our study, rabbit alveolar macrophages released TNF- $\alpha$, IL-6, IL-8, and IL-1 $\beta$ after stimulation with LukS-PV alone, which is a component of PVL. However, when LukF-PV was added to C5aR-bound LukS-PV on the macrophage surface to function as PVL, TNF- $\alpha$ was not released. Therefore, cytokine induction did not occur in alveolar macrophages due to PVL cytotoxicity.
Although there are differences in host species, toxin concentration, and stimulation time compared with those in our experiments, Magall et al. (26) also reported the release of IL- $1 \beta$ from human alveolar macrophages with PVL stimulation. Therefore, to elucidate the specific effect of PVL, it will be necessary to conduct cytotoxicity and cytokine measurements at various toxin concentrations and stimulation times. The time required for PVL pore formation on target cells and subsequent cytokine release should be investigated in greater detail. In a study related to cytokine release from target cells following LukS-PV stimulation, Zivkovic et al. (21) demonstrated that TLR2-mediated mechanisms could promote TNF- $\alpha$ and IL- 8 release independently of pore formation associated with C5aR. In-depth studies are required to evaluate multiple factors including TLR2 in addition to C5aR to further elucidate the mechanism of cytokine release by target cells.

In innate immunity against bacterial pneumonia, alveolar macrophages perform phagocytosis (27) and promote the migration of immune cells, including neutrophils, into the alveolar space by releasing chemokines and cytokines, such as TNF- $\alpha$ (28), IL-6 (29), IL-1 $\beta$ (30), and IL-8 (29,31). Based on our results, $\mathrm{PVL}$ is predicted to affect local immunity in the alveolar space as follows: (i) When LukS-PV acts alone, it 


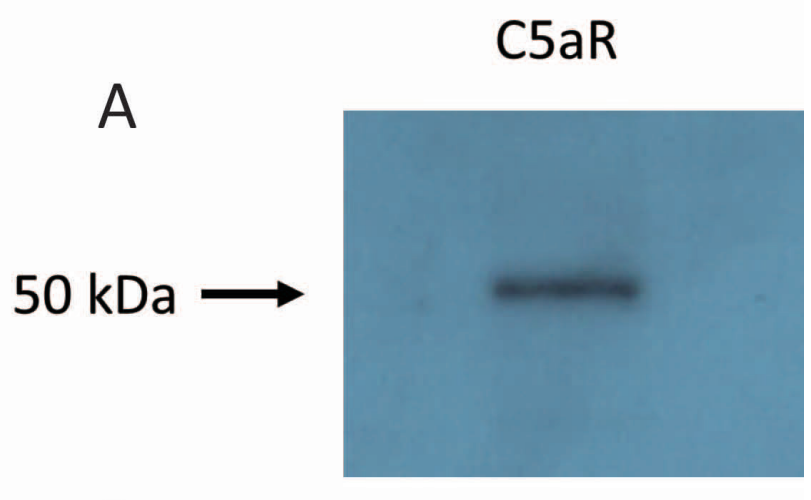

B

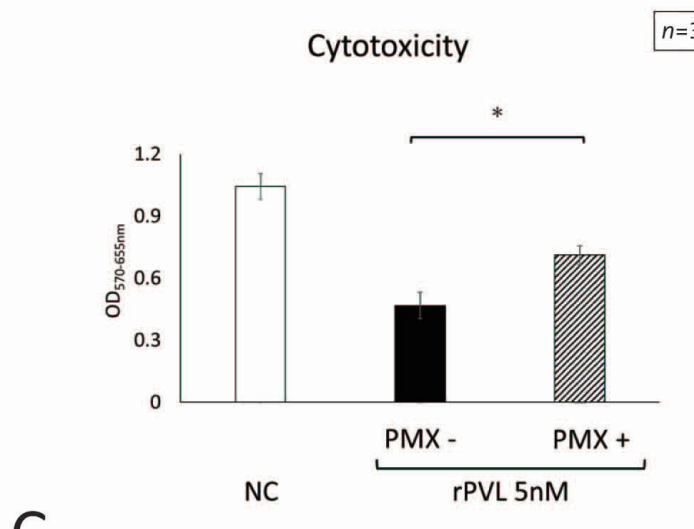

C

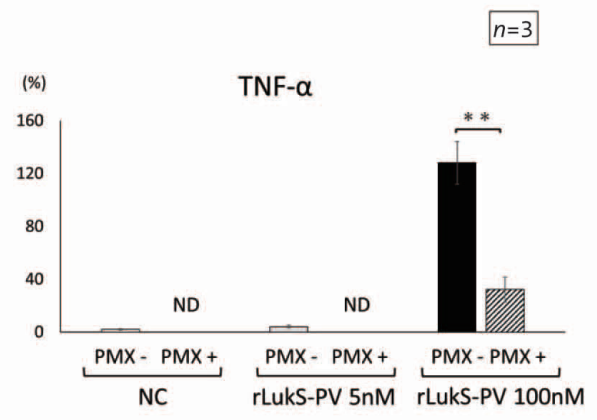

Fig. 4. (Color online) Confirmation of inhibition of cytotoxicity and suppression of cytokine using PMX205. (A) Expression of C5a receptor in alveolar macrophages was confirmed by western blotting. (B) Alveolar macrophages were pretreated with 100 $\mu \mathrm{M}$ PMX205 (PMX) for $15 \mathrm{~min}$, and then stimulated with $5 \mathrm{nM}$ rPVL (total concentration) for $24 \mathrm{~h}$; cytotoxicity was evaluated by the MTT assay. $n$ indicates the number of rabbits. ${ }^{*} P<0.05$. (C) Alveolar macrophages were pretreated with $100 \mu \mathrm{M}$ PMX for $15 \mathrm{~min}$ and then stimulated with 5 and $100 \mathrm{nM}$ rLukS-PV, PC (LPS $100 \mathrm{ng} / \mathrm{mL}$ ) for $24 \mathrm{~h}$, and TNF- $\alpha$ levels in the supernatant were measured by ELISA. Results represent the means \pm SE. $n$ indicates the number of rabbits. Significant differences were analyzed between toxin only group and PMX pretreated group by $t$-test. $* * P<0.01$.

promotes inflammatory cytokine release from alveolar macrophages through $\mathrm{C} 5 \mathrm{aR}$, which activates the innate immunity. (ii) However, when LukS-PV and LukF-PV bind to form PVL, C5aR-mediated cytotoxicity impairs phagocytosis and cytokine release, delays the removal of virulence factors, and prolongs infection (Fig. 5).

Diep et al. (32) established a rabbit model of necrotizing pneumonia using PVL-positive $S$. aureus
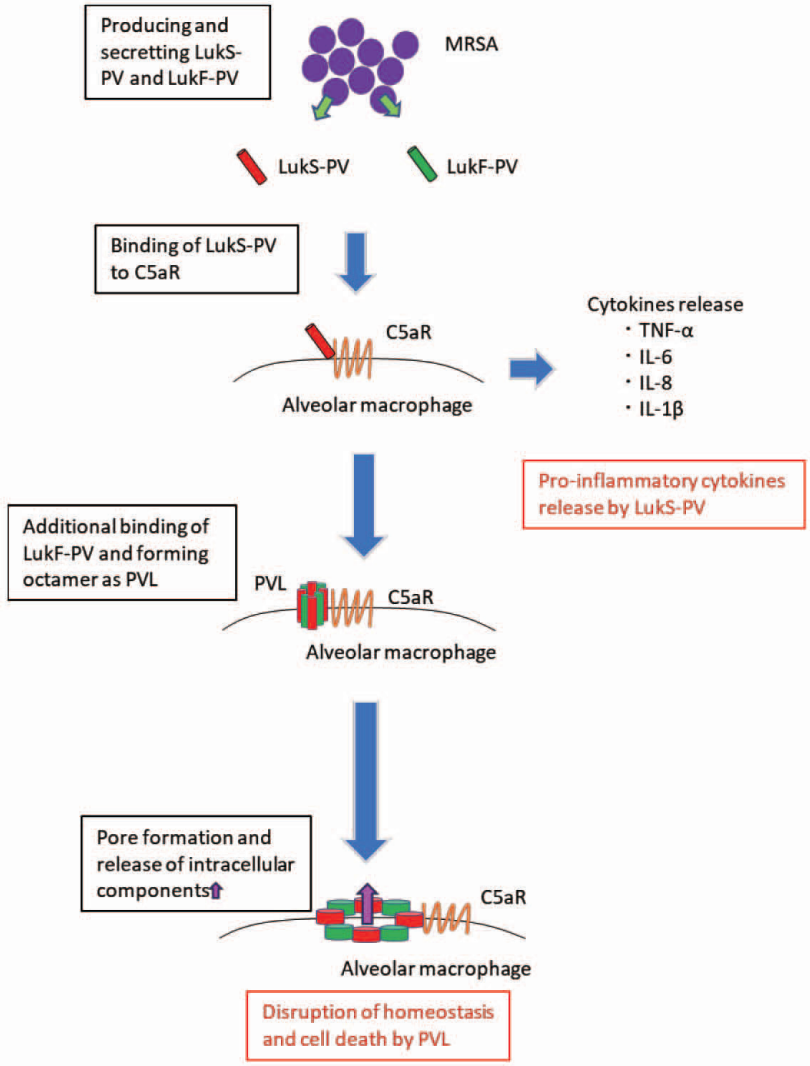

Fig. 5. (Color online) Proposed mechanism of action of PVL on alveolar macrophages. rLukS-PV alone induces C5aRmediated release of pro-inflammatory cytokines from alveolar macrophages. PVL forms pores in alveolar macrophages via $\mathrm{C} 5 \mathrm{aR}$ and results in cell death.

and reported the role of PVL in the alveolar space by assessing the levels of IL- 8 in the BALF and polymorphonuclear leukocyte infiltration in the lung tissue. Our findings suggest that alveolar macrophages may be involved in increasing the levels of IL- 8 in the alveolar space. We plan to isolate additional cell types such as neutrophils and alveolar epithelial cells, which are involved in local immunity in the alveolar space, to evaluate the effect of PVL on these cells and associated interactions.

In conclusion, we shed light on the inflammationinducing effect of PVL on alveolar macrophages, which play a central role in innate immunity. The results could be useful for understanding the mechanism of necrotizing pneumonia caused by PVL. In future studies, we will evaluate the effect of PVL on different cell types such as neutrophils and alveolar epithelial cells, which are involved in local immunity in the alveolar space, and associated interactions to further examine the mechanism of necrotizing pneumonia caused by PVL.

Acknowledgments We thank Dr. Masanori Yokoba for support with statistical analysis and Dr. Masaki Nakamura for support with ELISA. We would like to thank Editage (www.editage.com) for English language editing. 
Conflict of interest None to declear.

\section{REFERENCES}

1. Brumfitt W, Hamilton-Miller J. Methicillin-resistant Staphylococcus aureus. N Engl J Med. 1989;320:1188-96.

2. Keane CT, Cafferkey MT. Severe infections caused by methicillin-resistant Staphylococcus aureus. Eur J Clin Microbiol. 1983;2:299-302.

3. Buescher SE. Community-acquired methicillin-resistant Staphylococcus aureus in pediatrics. Curr Opin Pediatr. 2005; 17:67-70.

4. Peleg AY, Munckhof WJ. Fatal necrotising pneumonia due to community-acquired methicillin-resistant Staphylococcus aureus (MRSA). Med J.Aust. 2004;181:228-9.

5. Dufour P, Gillet Y, Bes M, et al. Community-acquired methicillinresistant Staphylococcus aureus infections in France: emergence of a single clone that produces Panton-Valentine leukocidin. Clin Infect Dis. 2002;35:819-24.

6. Yonezawa R, Kuwana $\mathrm{T}$, Kawamura $\mathrm{K}$, et al. Invasive community-acquired methicillin-resistant Staphylococcus aureus in a Japanese girl with disseminating multiple organ infection: a case report and review of Japanese pediatric cases. Case Rep Pediatr. 2015;2015:291025.

7. Francis JS, Doherty MC, Lopatin U, et al. Severe communityonset pneumonia in healthy adults caused by methicillin-resistant Staphylococcus aureus carrying the Panton-Valentine Leukocidin genes. Clin Infect Dis. 2005;40:1100-7.

8. Gonzalez BE, Martinez-Aguilar G, Hulten KG, et al. Severe staphylococcal sepsis in adolescents in the era of communityacquired methicillin-resistant Staphylococcus aureus. Pediatrics. 2005;115:642-8.

9. Nakagawa S, Taneike I, Mimura D, et al. Gene sequences and specific detection for Panton-Valentine leukocidin. Biochem Biophys Res Commun. 2005;328:995-1002.

10. Gillet Y, Issartel B, Vanhems P, et al. Association between Staphylococcus aureus strains carrying gene for Panton-Valentine leukocidin and highly lethal necrotizing pneumonia in young immunocompetent patients. Lancet. 2002;359:753-9.

11. Löffler B, Hussain M, Grundmeier M, et al. Staphylococcus aureus Panton-Valentine leukocidin is a very potent cytotoxic factor for human neutrophils. PLoS Pathog. 2010;6:e1000715.

12. Wu B, Zhang W, Huang J, et al. Effect of recombinant PantonValentine leukocidin in vitro on apoptosis and cytokine production of human alveolar macrophages. Can J Microbiol. 2010;56:229-35.

13. Gauduchon V, Sandra W, Gilles P, et al. Flow cytometric determination of Panton-Valentine leucocidin S component binding. Infect Immun. 2001;69:2390-5.

14. Oliveira D, Borges A, Simões M. Staphylococcus aureus toxins and their molecular activity in infectious diseases. Toxins (Basel). 2018;10:252.

15. Miles G, Movileanu L, Bayley H. Subunit composition of a bicomponent toxin: staphylococcal leukocidin forms an octameric transmembrane pore. Protein Sci. 2002;11:894-902.

16. Jayasinghe L, Bayley $\mathrm{H}$. The leukocidin pore: evidence for an octamer with four LukF subunits and four LukS subunits alternating around a central axis. Protein Sci. 2005;14:2550-61.

17. Aman MJ, Karauzum H, Bowden MG, et al. Structural model of the pre-pore ring-like structure of Panton-Valentine leukocidin: providing dimensionality to biophysical and mutational data. J Biomol Struct Dyn. 2010;28:1-12.

18. Spaan AN, Schiepers A, de Haas CJC, et al. Differential interaction of the staphylococcal toxins Panton-Valentine leukocidin and $\gamma$-hemolysin $\mathrm{CB}$ with human $\mathrm{C} 5$ a receptors. J Immunol. 2015;195:1034-43.

19. Spaan AN, Henry T, van Rooijen WJM, et al. The staphylococcal toxin Panton-Valentine leukocidin targets human $\mathrm{C} 5$ a receptors. Cell Host Microbe. 2013;13:584-94.

20. Holzinger D, Gieldon L, Mysore V, et al. Staphylococcus aureus Panton-Valentine leukocidin induces an inflammatory response in human phagocytes via the NLRP3 inflammasome. J Leukoc Biol. 2012;92:1069-81.

21. Zivkovic A, Sharif O, Stich K, et al. TLR 2 and CD14 mediate innate immunity and lung inflammation to staphylococcal PantonValentine leukocidin in vivo. J Immunol. 2011;186:1608-17.

22. March DR, Proctor LM, Stoermer MJ, et al. Potent cyclic antagonists of the complement $\mathrm{C} 5$ a receptor on human polymorphonuclear leukocytes: relationships between structures and activity. Mol Pharmacol. 2004;65:868-79.

23. Ma X, Chang W, Zhang C, et al. Staphylococcal Panton-Valentine leukocidin induces pro-inflammatory cytokine production and nuclear factor-kappa B activation in neutrophils. PLoS One. 2012;7:e34970.

24. Morito T, Oishi K, Yamamoto M, et al. Biphasic regulation of Fcreceptor mediated phagocytosis of rabbit alveolar macrophages by surfactant phospholipids. Tohoku J Exp Med. 2000;190:15-22.

25. Quinton LJ, Walkey AJ, Mizgerd JP. Integrative physiology of pneumonia. Physiol Rev. 2018;98:1417-64.

26. Perret M, Badiou C, Lina G, et al. Cross-talk between Staphylococcus aureus leukocidins-intoxicated macrophages and lung epithelial cells triggers chemokine secretion in an inflammasome-dependent manner. Cell Microbiol. 2012;14:101936.

27. Nagre N, Cong X, Pearson AC, et al. Alveolar macrophage phagocytosis and bacteria clearance in mice. J Vis Exp. 2019;145:10.3791/59088.

28. Soni S, Wilson MR, O'Dea KP, et al. Alveolar macrophagederived microvesicles mediate acute lung injury. Thorax. 2016;71:1020-9.

29. Larsson BM, Larsson K, Malmberg P, et al. Gram positive bacteria induce IL-6 and IL-8 production in human alveolar macrophages and epithelial cells. Inflammation. 1999;23:217-30.

30. Peiró T, Patel DF, Akthar S, et al. Neutrophils drive alveolar macrophage IL-1 $\beta$ release during respiratory viral infection. Thorax. 2018;73:546-56.

31. Miller EJ, Nagao S, Carr FK, et al. Interleukin-8 (IL-8) is a major neutrophil chemotaxin from human alveolar macrophages stimulated with staphylococcal enterotoxin A (SEA). Inflamm Res. 1996;45:386-92.

32. Diep BA, Chan L, Tattevin P, et al. Polymorphonuclear leukocytes mediate Staphylococcus aureus pantonvalentine leukocidin-induced lung inflammation and injury. Proc Natl Acad Sci U S A. 2010;107:5587-92. 